\title{
ALMOST EXACTNESS IN NORMED SPACES
}

\author{
ROBIN HARTE
}

\begin{abstract}
Almost exactness" is defined for pairs of bounded operators between normed spaces.
\end{abstract}

Introduction. If $T: X \rightarrow Y$ and $S: Y \rightarrow Z$ are linear operators between linear spaces for which

$$
S^{-1}(0)=T(X)
$$

then the pair $(S, T)$ is sometimes described as "exact". For bounded operators between normed spaces the proper definition is more elusive. In this note we offer definitions of "exactness" and "almost exactness" in $\S 1$, which amount to simultaneous generalizations of boundedness below and respectively openness and almost openness; we show how they are related to the fundamental subspace-quotient sequence, and we demonstrate that almost exact pairs come in open sets. In $\S 2$ we see how almost exactness can be expressed using duality, "enlargement", and completion, and in $\S 3$ we address ourselves to a boundary problem. It turns out that, provided the spaces are complete, "almost exactness" reduces to condition (0.1) together with an additional closed range condition, and that "almost exactness" in the incomplete case reduces to the same condition for the completed operators. In a sense therefore most of what we have to say can be recovered from earlier work. We believe however that our point of view helps to bring out some of the "resonances" latent in the usual form of the exactness condition for complete spaces.

1. Suppose $T \in B L(X, Y)$ and $S \in B L(Y, Z)$ are bounded linear operators between normed spaces; then we shall call the pair $(S, T)$ (left, right) invertible if there are $T^{\prime} \in B L(Y, X)$ and $S^{\prime} \in B L(Z, Y)$ for which

$$
S^{\prime} S+T T^{\prime}=I
$$

and (left, right) one-one if there is inclusion

$$
S^{-1}(0) \subseteq \operatorname{cl}(T X) \text {. }
$$

When also

$$
S T=0,
$$

then (1.0.1) and (1.0.2) represent respectively the strongest and the weakest possible kinds of exactness. We shall write

$$
B L(X, Y, Z)=\{(S, T) \in B L(Y, Z) \times B L(X, Y): S T=0\}
$$

for the closed subset of chains $(S, T)$ in $B L(Y, Z) \times B L(X, Y)$.

Received by the editors January 17, 1986 and, in revised form, April 15, 1986.

1980 Mathematics Subject Classification (1985 Revioion). Primary 47A99; Secondary 46B99.

Key words and phrases. Exact, almost exact, (left, right)-invertible, nonsingular, enlargement, completion. 
The concept of exactness appropriate to normed spaces is more subtle:

1.1 Definition. The pair $(S, T) \in B L(Y, Z) \times B L(X, Y)$ will be called almost (left, right) nonsingular if there are $k, k^{\prime}>0$ for which, for each $y \in Y$,

$$
y \in \operatorname{cl}_{Y}\left(\operatorname{Disc}_{Y}\left(0 ; k^{\prime}\|S y\|\right)+T \operatorname{Disc}_{X}(0 ; k\|y\|)\right),
$$

(left, right) nonsingular if, for each $y \in Y$,

$$
y \in \operatorname{Disc}_{Y}\left(0 ; k^{\prime}\|S y\|\right)+T \operatorname{Disc}_{X}(0 ; k\|y\|),
$$

and strictly (left, right) nonsingular if in addition

$$
S(Y)=\operatorname{cl}_{Z}(S Y) \text {. }
$$

If in addition $(S, T)$ is a chain, then it will be called almost exact, exact, or strictly exact.

Necessary and sufficient for (1.1.1) is that for each $y \in Y$ there should be a sequence $\left(x_{n}\right)$ in $X$ for which

$$
\lim _{n} \sup \left\|y-T x_{n}\right\| \leq k^{\prime}\|S y\| \quad \text { and } \sup _{n}\left\|x_{n}\right\| \leq k\|y\| ;
$$

for (1.1.2) we should have (1.1.4) with a constant sequence $x_{n}=x$. For example if $T=0$ then each of (1.1.1) and (1.1.2) reduces to the condition that $S$ is bounded below; if instead $S=0$ then (1.1.1) is the condition that $T$ is almost open [7], and (1.1.2) the condition that $T$ is open. In general there is the implication

$$
(1.0 .1) \Rightarrow((1.1 .3) \text { and }(1.1 .2)) \Rightarrow(1.1 .2) \Rightarrow(1.1 .1) \Rightarrow(1.0 .2) \text {; }
$$

if in particular $T=T T^{\prime \prime} T$ and $S=S S^{\prime \prime} S$ have "generalized inverses" then [5, Lemma $2 ; 8$, Theorem 1.6] (1.0.2) $\Rightarrow(1.0 .1)$, so all these conditions coalesce.

Perhaps the most fundamental example of an almost exact pair is the subspacequotient sequence; (1.1.1) holds with $k=2$ and $k^{\prime}=1$ when $X=W \subseteq Y$ with natural injection $T: X \rightarrow Y$ and quotient mapping $S: Y \rightarrow Z=Y / \operatorname{cl}(W)$ :

1.2 THEOREM. If $W$ is a subspace of $Y$, then for each $y \in Y$ there is a sequence $\left(w_{n}\right)$ in $W$ for which

$$
\left\|y-w_{n}\right\| \rightarrow \operatorname{dist}(y, W) \quad \text { and } \quad\left\|w_{n}\right\| \leq\|y\|+\operatorname{dist}(y, W) \leq 2\|y\| .
$$

If $W$ is closed and $k^{\prime}>1$, then for each $y \in Y$ there is $w \in W$ for which

$$
\|y-w\| \leq k^{\prime} \operatorname{dist}(y, W) \quad \text { and } \quad\|w\| \leq\|y\|+\operatorname{dist}(y, W) \leq 2\|y\| .
$$

ProOF. By definition of $\operatorname{dist}(y, W)$ there is $\left(w_{n}^{\prime}\right)$ in $W$ for which

$$
\left\|y-w_{n}^{\prime}\right\| \leq \operatorname{dist}(y, W)+(1 / n)\|y\| \quad \text { for each } n \in \mathbb{N},
$$

which implies

$$
\left\|w_{n}^{\prime}\right\| \leq\|y\|+\left\|y-w_{n}^{\prime}\right\| \leq \operatorname{dist}(y, W)+(1+1 / n)\|y\|
$$

thus (1.2.1) holds with

$$
w_{n}=(n /(n+1)) w_{n}^{\prime} .
$$

Whether or not $W$ is closed, if $y \in W$ then (1.2.2) holds with $w=y$, while if $y \notin \operatorname{cl}(W)$ so that $\operatorname{dist}(y, W)>0$ we can replace (1.2.3) by

$$
\left\|y-w_{n}^{\prime}\right\| \leq \operatorname{dist}(y, W)+(1+1 / n) \operatorname{dist}(y, W) \quad \text { for each } n \in \mathbb{N} \text {. }
$$


Now if we define $w_{n}$ by the formula (1.2.4) then the second part of (1.2.1) still holds, while

$\left\|y-w_{n}\right\| \leq\left\|y-w_{n}^{\prime}\right\|+\left\|w_{n}^{\prime}\right\| /(n+1) \leq(1+1 / n)(1+1 /(n+1)) \operatorname{dist}(y, W)+\|y\| /(n+1) ;$

thus (1.2.2) holds if we take

$$
w=w_{N} \quad \text { with }(3+\|y\| / \operatorname{dist}(y, W)) / N \leq k^{\prime}-1 .
$$

Thus if $W=\operatorname{cl}(W)$ is closed, then we can arrange (1.2.2) for every $y \in Y$.

Theorem 1.2 is a sort of "anti Riesz lemma": the special case in which $Y=\operatorname{cl}(W)$ was part of Theorem 1.3 of [7]. Edward Bach of Trinity College, Dublin has shown (unpublished communication) that we cannot always get $\left\|w_{n}\right\| \leq\|y\|$ in (1.2.1). Theorem 1.2 enables us to break the almost exactness condition into two pieces:

1.3 THEOREM. Necessary and sufficient for a chain $(S, T) \in B L(X, Y, Z)$ to be almost exact is that there are $h>0$ and $h^{\prime}>0$ for which, for each $y \in Y$,

$$
\operatorname{dist}(y, T X) \leq h^{\prime}\|S y\|
$$

and

$$
S y=0 \Rightarrow y \in \operatorname{cl}_{Y}\{T x:\|x\| \leq h\|y\|\} .
$$

Necessary and sufficient for $(S, T)$ to be exact is that in addition

$$
S y=0 \Rightarrow y \in\{T x:\|x\| \leq h\|y\|\} .
$$

PROOF. If (1.1.1) holds then, whether or not $(S, T)$ satisfies the chain condition (1.0.3), both (1.3.1) and (1.3.2) follow at once, with $h=k$ and $h^{\prime}=k^{\prime}$; if (1.1.2) holds then so also does (1.3.3). Conversely if $y \in Y$ is arbitrary then (1.2.1) gives a sequence $\left(w_{n}\right)$ in $Y$ for which

$$
\left\|S w_{n}\right\|=0, \quad\left\|y-w_{n}\right\| \rightarrow \operatorname{dist}\left(y, S^{-1}(0)\right), \quad \text { and } \quad\left\|w_{n}\right\| \leq 2\|y\| .
$$

By (1.3.2) there is a sequence $\left(x_{n}\right)$ in $X$ for which

$$
\left\|w_{n}-T x_{n}\right\| \rightarrow 0 \quad \text { and } \quad\left\|x_{n}\right\| \leq h\left\|w_{n}\right\| .
$$

Together these give

$$
\left\|y-T x_{n}\right\| \rightarrow \operatorname{dist}\left(y, S^{-1}(0)\right) \quad \text { and } \quad\left\|x_{n}\right\| \leq 2 h\|y\| .
$$

If we now remember that $S T=0$ and then apply (1.3.1) we have also

$$
\operatorname{dist}\left(y, S^{-1}(0)\right) \leq \operatorname{dist}(y, T X) \leq h^{\prime}\|S y\| .
$$

Thus (1.1.1) holds with $k=2 h$ and $k^{\prime}=h^{\prime}$. At the same time (1.2.2) gives, since $S^{-1}(0)$ is closed, $w \in Y$ for which

$$
S w=0, \quad\|y-w\| \leq(1+\varepsilon) \operatorname{dist}(y, W), \quad \text { and } \quad\|w\| \leq 2\|y\|,
$$

and then if (1.3.3) holds there is $x \in X$ for which

$$
w=T x \quad \text { and } \quad\|x\| \leq h\|w\| .
$$

Using this with the argument above shows that (1.1.2) holds with $k=2 h$ and $k^{\prime}=(1+\varepsilon) h^{\prime}$.

We are unable to settle whether or not Theorem 1.3 can be extended to pairs $(S, T)$ for which $S T \neq 0$. When $X, Y$ and $Z$ are complete then Theorem 1.3 and 
the open mapping theorem combine to say that a chain $(S, T) \in B L(X, Y, Z)$ will be almost exact, equivalently exact, iff

$$
T(X)=S^{-1}(0) \quad \text { and } \quad S(Y)=\operatorname{cl}_{Z}(S Y)
$$

thus if the spaces are complete then all three conditions in Definition 1.1 are equivalent.

Almost exact pairs come in open sets:

1.4 THEOREM. If $X, Y$, and $Z$ are normed spaces then

$$
\{(S, T) \in B L(X, Y, Z):(S, T) \text { is almost exact }\}
$$

is an open subset of the space $B L(X, Y, Z)$.

ProOF. Suppose that $k, k^{\prime}>0$ satisfy (1.1.1): then if $\left(S^{\prime}, T^{\prime}\right) \in B L(X, Y, Z)$ satisfies

$$
k\left\|T^{\prime}-T\right\|+k^{\prime}\left\|S^{\prime}-S\right\|+\varepsilon=\delta<1 \text { with } \varepsilon>0,
$$

we claim that (1.3.1) and (1.3.2) hold for $\left(S^{\prime}, T^{\prime}\right)$, with $h=k /(1-\delta)$ and $h^{\prime}=$ $k^{\prime} /(1-\delta)$. If $y \in Y$ then (1.1.4) gives $x_{1} \in X$ for which

$$
\left\|y-T x_{1}\right\| \leq k^{\prime}\|S y\|+\varepsilon\|y\| \quad \text { and } \quad\left\|x_{1}\right\| \leq k\|y\|
$$

and hence

$$
\begin{aligned}
\left\|y-T^{\prime} x_{1}\right\| & \leq\left\|\left(T^{\prime}-T\right) x_{1}\right\|+k^{\prime}\|S y\|+\varepsilon\|y\| \\
& \leq k^{\prime}\left\|S^{\prime} y\right\|+\left\|T^{\prime}-T\right\|\left\|x_{1}\right\|+k^{\prime}\left\|S^{\prime}-S\right\|\|y\|+\varepsilon\|y\|,
\end{aligned}
$$

so that, with $y_{1}=y-T^{\prime} x_{1}$,

$$
\begin{aligned}
& y=y_{1}+T^{\prime} x_{1} \quad \text { with } S^{\prime} y=S^{\prime} y_{1}, \\
& \left\|y_{1}\right\| \leq k^{\prime}\left\|S^{\prime} y\right\|+\delta\|y\| \text { and }\left\|x_{1}\right\| \leq k\|y\| .
\end{aligned}
$$

Iteration gives sequences $\left(y_{n}\right)$ in $Y$ and $\left(x_{n}\right)$ in $X$ for which

$$
\begin{gathered}
y=y_{n}+T^{\prime}\left(x_{1}+x_{2}+\cdots+x_{n}\right) \quad \text { with } S^{\prime} y_{n}=S^{\prime} y, \\
\left\|y_{n}\right\| \leq k^{\prime}\left(1+\delta+\cdots+\delta^{n-1}\right)\left\|S^{\prime} y\right\|+\delta^{n}\|y\| \leq h^{\prime}\left\|S^{\prime} y\right\|+\delta^{n}\|y\|,
\end{gathered}
$$

and

$$
\begin{aligned}
\| x_{1} & +x_{2}+\cdots+x_{n} \| \\
& \leq k k^{\prime}\left(n+(n-1) \delta+\cdots+\delta^{n-1}\right)\left\|S^{\prime} y\right\|+k\left(1+\delta+\cdots+\delta^{n-1}\right)\|y\| \\
& \leq n h^{\prime \prime}\left\|S^{\prime} y\right\|+h\|y\| .
\end{aligned}
$$

Here $h=k /(1-\delta), h^{\prime}=k^{\prime} /(1-\delta)$, and $h^{\prime \prime}=k k^{\prime} /(1-\delta)$. Now (1.4.5) and (1.4.6) show that $\left(S^{\prime}, T^{\prime}\right)$ satisfies (1.3.1), where no control is needed on the size of $\left\|x_{1}+x_{2}+\cdots+x_{n}\right\|$, and together with (1.4.7) show that $\left(S^{\prime}, T^{\prime}\right)$ satisfies (1.3.2), since if $S^{\prime} y=0$ then $\left\|x_{1}+x_{2}+\cdots+x_{n}\right\| \leq h\|y\|$.

Our proof of Theorem 1.4 closely follows the original argument of Joseph Taylor [12, Lemma 2.1], which assumes that the spaces $X, Y$, and $Z$ are complete and works with the condition (1.3.5); the same argument is given by Vasilescu [13, Lemma 2.1], and a quantitative version is given by Potra and Ptak [11, Chapter 10, Proposition 10]. We might observe however that in each case the pair $(S, T)$ is assumed to satisfy $(1.3 .6)$, while the pair $\left(S^{\prime}, T^{\prime}\right)$ is only proved to satisfy $(0.1)$. 
The reader is invited to see whether by sufficient ingenuity he can bypass Theorem 1.3 in the proof of Theorem 1.4. No amount of such ingenuity however will remove the assumption that the pairs $\left(S^{\prime}, T^{\prime}\right)$ are chains: If for example $X=Y=Z=C^{2}$ and [5, Example 2]

$$
S=\left(\begin{array}{ll}
1 & 0 \\
0 & 0
\end{array}\right) \text { and } T=\left(\begin{array}{ll}
0 & 0 \\
0 & 1
\end{array}\right)
$$

then $(S, T) \in B L(X, Y, Z)$ is (left, right) invertible in the sense of (1.0.1), but can be approximated by the pairs $\left(S_{t}, T_{t}\right)$ with $0 \neq t \in \mathrm{C}$ given by

$$
S_{t}=\left(\begin{array}{ll}
1 & t \\
0 & 0
\end{array}\right) \quad \text { and } T_{t}=\left(\begin{array}{ll}
0 & t \\
0 & 1
\end{array}\right)
$$

which are not even (left, right) one-one in the sense of (1.0.2).

2. Exactness and almost exactness can be described in the dual spaces, in the "enlargements", and in the completions. If we write

$$
X^{\dagger}=B L(X, C)
$$

for the dual of a normed space $X$, and

$$
T^{\dagger}: Y^{\dagger} \rightarrow X^{\dagger}
$$

for the operator induced by $T \in B L(X, Y)$, then it follows from Theorem 1.3 and the usual Hahn-Banach arguments [7, (2.3.3) and (2.3.4)] that

$$
(S, T) \text { almost exact } \Leftrightarrow\left(T^{\dagger}, S^{\dagger}\right) \text { almost exact. }
$$

Less predictably,

2.1 THEOREM. Necessary and sufficient for a chain $(S, T) \in B L(X, Y, Z)$ to be almost exact is that there are $k, k^{\prime}>0$ for which, for each $y \in Y, g \in Y^{\dagger}$,

$$
|g(y)| \leq k\|g T\|\|y\|+k^{\prime}\|g\|\|S y\| .
$$

PROOF. Whether or not $(S, T)$ is a chain, if (1.1.4) holds then for arbitrary $y \in Y$, arbitrary $W$, and arbitrary $V \in B L(Y, W)$ we have

$$
\|V y\| \leq\left\|V\left(y-T x_{n}\right)\right\|+\left\|V T x_{n}\right\| \leq\|V\|\left\|y-T x_{n}\right\|+\|V T\|\left\|x_{n}\right\|
$$

and hence, taking limsup $\sup _{n}$,

$$
\|V y\| \leq k\|V T\|\|y\|+k^{\prime}\|V\|\|S y\| .
$$

Specializing to $W=C$ gives (2.1.1). For the converse we use Theorem 1.3: if (1.3.1) fails then there is $\left(y_{n}\right)$ in $Y$ for which

$$
\left\|y_{n}\right\|=1, \quad \operatorname{dist}\left(y_{n}, T X\right) \geq \frac{1}{2}, \quad \text { and } \quad\left\|S y_{n}\right\| \rightarrow 0,
$$

and then by the Hahn-Banach theorem there is $\left(g_{n}\right)$ in $Y^{\dagger}$ for which

$$
\left\|g_{n}\right\|=\left|g_{n}\left(y_{n}\right)\right|=1 \text { and }\left\|g_{n} T\right\|=0 .
$$

Together these violate (2.1.1). If instead (1.3.2) fails then, using the Hahn-Banach separation theorem, there is $\left(g_{n}\right)$ in $Y^{\dagger}$ for which

$$
\left\|g_{n}\right\|_{S^{-1}(0)}=1 \text { and }\left\|g_{n} T\right\| \rightarrow 0,
$$


and then $\left(y_{n}\right)$ in $Y$ for which

$$
\left\|y_{n}\right\|=1, \quad S y_{n}=0, \quad \text { and } \quad\left|g_{n}\left(y_{n}\right)\right| \geq \frac{1}{2}
$$

Again these conditions together violate (2.1.1).

It is clear from (2.1.2) that if $(S, T)$ is almost (left, right) nonsingular then

$$
\|V U\| \leq k\|V T\|\|U\|+k^{\prime}\|V\|\|T U\|
$$

for arbitrary $U \in B L\left(W^{\prime}, X\right)$ and $V \in B L(Y, W)$.

We recall from [7] the enlargement

$$
\mathbf{Q}(X)=l_{\infty}(X) / c_{0}(X)
$$

of a normed space $X$, together with the operator

$$
\mathbf{Q}(T): \mathbf{Q}(X) \rightarrow \mathbf{Q}(Y)
$$

induced by $T \in B L(X, Y)$; note particularly the norm of a coset

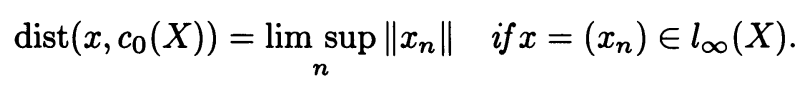

2.2 TheOREM. If $(S, T) \in B L(X, Y, Z)$ is a chain then the following are equivalent:

$$
\begin{gathered}
(\mathbf{Q}(S), \mathbf{Q}(T)) \text { is (left,right) one-one; } \\
(S, T) \text { is almost exact; } \\
(\mathbf{Q}(S), \mathbf{Q}(T)) \text { is exact. }
\end{gathered}
$$

Proof. Whether or not $(S, T)$ is a chain, if (2.2.2) holds then by (1.1.4) there is for each sequence $y=\left(y_{n}\right)$ in $Y$ a sequence $x=\left(x_{n}\right)$ in $X$ for which

$$
\left\|y_{n}-T x_{n}\right\| \leq k^{\prime}\left\|S y_{n}\right\|+(1 / n)\left\|y_{n}\right\| \text { and }\left\|x_{n}\right\| \leq k\left\|y_{n}\right\| \text {. }
$$

Applying this with $y \in l_{\infty}(Y)$ shows that $(\mathbf{Q}(S), \mathbf{Q}(T))$ satisfies condition (1.1.2), as required by (2.2.3). Conversely if (2.2.2) fails and we assume that $S T=0$ then by Theorem 1.3 either (1.3.1) or (1.3.2) must fail: in each case we claim there is a sequence $y=\left(y_{n}\right)$ in $Y$ for which

$$
y \in l_{\infty}(Y) \text { and } S y \in c_{0}(Y)
$$

and

$$
x \in l_{\infty}(X) \Rightarrow \operatorname{dist}\left(y-T x, c_{0}(Y)\right) \geq \frac{1}{2} .
$$

If (1.3.1) fails then we may choose $y$ as in (2.1.3). If instead (1.3.2) fails then we may choose $y$ as in (2.1.6), with $g=\left(g_{n}\right)$ as in (2.1.5): now for arbitrary $x \in l_{\infty}(X)$ we argue

$$
\begin{aligned}
\left\|y_{n}-T x_{n}\right\| & =\left\|g_{n}\right\|\left\|y_{n}-T x_{n}\right\| \geq\left|g_{n}\left(y_{n}-T x_{n}\right)\right| \\
& \geq\left|g_{n}\left(y_{n}\right)\right|-\left\|g_{n} T\right\|\left\|x_{n}\right\| \geq \frac{1}{2}-\|x\|_{\infty}\left\|g_{n} T\right\|
\end{aligned}
$$

and hence

$$
\limsup _{n}\left\|y_{n}-T x_{n}\right\| \geq \underset{n}{\lim \sup }\left(\frac{1}{2}-\|x\|_{\infty}\left\|g_{n} T\right\|\right)=\frac{1}{2} .
$$

The last part of the argument is suggested by the proof of part of Lemma 2.1 of [1]. If we take $S=0$ in Theorem 2.2 we get a simplified version of the proof 
of Theorem 1.1 of [9]. One by-product of Theorem 2.2 is that almost exactness in incomplete spaces can be tested in their completions: here we recall

$$
X^{\sim}=c(X) / c_{0}(X) \subseteq \mathbf{Q}(X)
$$

the familiar completion of $X$, together with the operator

$$
T^{\sim}: X^{\sim} \rightarrow Y^{\sim}
$$

induced by $T \in B L(X, Y)$.

2.3 THEOREM. If $(S, T) \in B L(X, Y, Z)$ is a chain between normed spaces then the following are equivalent:

$$
\begin{gathered}
(S, T) \text { is almost exact; } \\
\left(S^{\sim}, T^{\sim}\right) \text { is almost exact; } \\
\left(S^{\sim}, T^{\sim}\right) \text { is strictly exact; } \\
T^{\sim}\left(X^{\sim}\right)=S^{-1}(0) \text { and } S^{\sim}\left(Y^{\sim}\right)=\operatorname{cl}\left(S^{\sim} Y^{\sim}\right) .
\end{gathered}
$$

ProOF. It is a special case [7, Theorem 4.2] of Theorem 2.2 that

$$
\mathbf{Q}(X) \cong \mathbf{Q}\left(X^{\sim}\right)
$$

thus Theorem 2.2 itself shows that (2.3.1) and (2.3.2) are equivalent. The equivalence of (2.3.2), (2.3.3), and (2.3.4), using the open mapping theorem, is our remark (1.3.6).

One consequence of (2.3.5) is that $\mathbf{Q}(X)$ is a!ways complete, whether or not the space $X$ is. This gives a further strengthening of Theorem 2.2: if $(S, T)$ satisfies (2.2.2) then also

$$
(\mathbf{Q}(S), \mathbf{Q}(T)) \text { is strictly exact. }
$$

3. We conclude with a discussion of a problem suggested by [7] and the boundary result of Cho and Tagachi $[\mathbf{3}, \mathbf{4}]$ :

3.1 Problem. If $(S, T)$ and $\left(S_{n}, T_{n}\right)$ in $B L(X, Y, Z)$ satisfy

$$
(S, T) \text { almost exact, }
$$

$$
S_{n} \text { dense and } T_{n}^{\dagger} \text { dense, }
$$

and

$$
\left\|S_{n}-S\right\|+\left\|T_{n}-T\right\| \rightarrow 0 \quad \text { as } n \rightarrow \infty,
$$

does it follow that

$$
S \text { dense and } T^{\dagger} \text { dense? }
$$

As an attempt to prove this, suppose that $z \in Z$ is arbitrary and find $\left(y_{n}\right)$ in $Y$ and then $\left(x_{n}\right)$ in $X$ for which $\left\|z-S_{n} y_{n}\right\| \rightarrow 0$ and

$$
\left\|y_{n}-T x_{n}\right\| \leq k^{\prime}\left\|S y_{n}\right\|+(1 / n)\|z\| \quad \text { with }\left\|x_{n}\right\| \leq k\left\|y_{n}\right\| .
$$


Now

$$
\begin{aligned}
\left\|z-S y_{n}\right\| \leq & \left\|z-S_{n} y_{n}\right\|+\left\|S_{n}-S\right\|\left\|y_{n}\right\| \\
\leq & \left\|z-S_{n} y_{n}\right\|+\left\|S_{n}-S\right\|\left\|y_{n}-T x_{n}\right\|+\left\|\left(S_{n}-S\right) T x_{n}\right\| \\
\leq & \left\|z-S_{n} y_{n}\right\|+\left\|S_{n}-S\right\|\left(k^{\prime}\left\|S y_{n}\right\|+(1 / n)\|z\|\right)+\left\|\left(S_{n}-S\right) T x_{n}\right\| \\
\leq & \left\|z-S_{n} y_{n}\right\|+\left\|S_{n}-S\right\| k^{\prime}\left\|S y_{n}-z\right\| \\
& +\left\|S_{n}-S\right\|\left(k^{\prime}+(1 / n)\right)\|z\|+\left\|\left(S_{n}-S\right) T x_{n}\right\|,
\end{aligned}
$$

giving

(3.1.6) $\left(1-k^{\prime}\left\|S_{n}-S\right\|\right)\left\|z-S y_{n}\right\| \leq\left\|S_{n}-S\right\|\left(k^{\prime}+(1 / n)\right)\|z\|+\left\|\left(S_{n}-S\right) T x_{n}\right\|$.

Our lack of control of the last term on the right-hand side stands between us and the result. In the special case $T=0$ this reduces to the proof of Theorem 1.3 of [7]. When $X, Y$, and $Z$ are Hilbert spaces then we can actually reduce the whole problem to this special case: observe $[4,10]$ that $(3.1 .1)$ is equivalent to

$$
S^{*} S+T T^{*}=\operatorname{row}\left(S^{*}, T\right) \operatorname{col}\left(S, T^{*}\right) \text { invertible, }
$$

and that similarly (3.1.4) is equivalent to

$$
\left(\begin{array}{cc}
S S^{*} & 0 \\
0 & T^{*} T
\end{array}\right)=\operatorname{col}\left(S, T^{*}\right) \operatorname{row}\left(S^{*}, T\right) \quad \text { one-one and dense. }
$$

Thus the assumptions of Problem 3.1 are that the operator $\operatorname{col}\left(S, T^{*}\right): Y \rightarrow Z \times X$ is bounded below, and can be approximated by the operators $\operatorname{col}\left(S_{n}, T_{n}^{*}\right): Y \rightarrow Z \times X$ which have dense range: then by Theorem 1.3 of $[7]$ it follows that $\operatorname{col}\left(S, T^{*}\right)$ is also dense, and hence almost open.

If we strengthen the assumptions in Problem 3.1, assuming that the chains $\left(S_{n}, T_{n}\right)$ form "short exact sequences", and use Theorem 2.3 to reduce to complete spaces, then an affirmative solution would seem to be contained in the indexstability results of Vasilescu and Albrecht $[13,2]$ : their arguments deal with unbounded operators, and use gap theory.

ADDED IN PROOF. A result dual to Probem 3.1 is obtained for complete spaces by Wrobel [14, Lemma 2.4].

\section{REFERENCES}

1. E. Albrecht and R. D. Mehta, Some remarks on local spectral theory, J. Operator Theory 12 (1984), 285-317.

2. E. Albrecht and F.-H. Vasilescu, Stability of the index of a semi-Fredholm complex of Banach spaces, J. Funct. Anal. (to appear); summarized in Operator Theory: Advances and Applications, vol. 11, Birkhäuser-Verlag, 1983, pp. 15-39.

3. M. Cho and M. Tagachi, Boundary of Taylor's joint spectrum, Rev. Roumaine Math. Pures Appl. 27 (1982), 863-866.

4. R. E. Curto, Connections between Harte and Taylor spectra, Rev. Roumaine Math. Pures Appl. 31 (1986).

5. R. E. Harte, A problem of mixed interpolation, Math. Z. 143 (1975), 149-153.

6. , Invertibility, singularity and Joseph L. Taylor, Proc. Roy. Irish Acad. Sect. A 81 (1981), 71-79.

7. __ Almost open mappings between normed spaces, Proc. Amer. Math. Soc. 90 (1984), 243-249.

8. , Fredholm, Weyl and Browder theory, Proc. Roy. Irish Acad. Sect. A 85 (1986), 151-176.

9. R. E Harte and M. Mathieu, Enlargements of almost open mappings, Proc. Amer. Math. Soc. 96 (1986), 247-248. 
10. R. E. Harte and M. Ó Séarcóid, Positive elements and the $B^{*}$ condition, Math. Z. 193 (1986), 1-9.

11. F. A. Potra and V. Ptak, Nondiscrete induction and interative processes, Research Notes in Math., no. 103, Pitman, New York, 1984.

12. J. L. Taylor, A joint spectrum for several commuting operators, J. Funct. Anal. 6 (1970), 172-191.

13. F.-H. Vasilescu, Stability of the index of a complex of Banach spaces, J. Operator Theory 2 (1979), 247-275.

14. V. Wrobel, The boundary of Taylor's joint spectrum for two commuting Banach space operators, Studia Math. (to appear).

Department of Mathematics, University College, Cork, IREland 